\title{
Triadic resonances driven by thermal convection in a rotating sphere
}

\author{
Yufeng $\operatorname{Lin}^{1} \dagger$ \\ ${ }^{1}$ Department of Earth and Space Sciences, Southern University of Science and Technology, \\ Shenzhen 518055, China
}

(Received $\mathrm{xx}$; revised $\mathrm{xx}$; accepted $\mathrm{xx}$ )

We report for the first time on triadic resonances in a rotating convection system. Using direct numerical simulations, we find that convective modes in a rotating spherical fluid can excite a pair of inertial modes whose frequencies and wavenumbers match the triadic resonance conditions. Depending on the structures of the convective modes, triadic resonances can lead to the growth of either a pair of modes with lower frequencies and wavenumbers, or a pair of modes with higher frequencies and wavenumbers, providing a possible mechanism for the bi-directional energy cascade. Increased thermal forcing leads to fully developed turbulence, which also exhibits wave-like motions, and is reminiscent of the energy spectrum of inertial wave turbulence. Our results suggest that the interaction of inertial waves plays an important role in rotating convection, which is of great importance in understanding the dynamics of planetary and stellar interiors.

\section{Introduction}

Fluid flows in stars and planets, which are typically subject to rotation, can be driven by internal thermal forcing (Spiegel 1971; Aurnou et al. 2015; Jones 2015) and/or by external mechanical forcings due to the gravitational coupling with orbital companions (Le Bars et al. 2015; Ogilvie 2014). Despite the common rotational constraint, thermal convection and mechanically driven flows are traditionally treated as two separate branches in rotating fluid dynamics (Chandrasekhar 1961; Greenspan 1968).

Owing to the periodicity of orbital motions, mechanical forcings such as precession (Hollerbach \& Kerswell 1995; Noir et al. 2001), libration (Aldridge \& Toomre 1969) and tides (Ogilvie \& Lin 2004) can drive oscillatory motions in rotating fluids, known as inertial modes or inertial waves restored by the Coriolis force (Greenspan 1968). On the other hand, it has been long recognized that rotating convection close to the onset is in the form of quasi-steady columnar rolls (Busse 1970; Jones et al. 2000). At low Prandtl number $(\operatorname{Pr}<1)$, however, it was found that the onset of convection can be in the form of an oscillatory inertial mode at leading order (Zhang 1994), suggesting that rotating convection and mechanically driven rotating flows are closely related. Indeed, a recent monograph by Zhang \& Liao (2017) provided a set of unified asymptotic theories for the linear onset of convection and linear responses to mechanical forcings in rotating fluids based on an expansion of inertial waves. In this paper, we show that some aspects of the nonlinear dynamics of rotating convection can be understood within the framework of inertial wave interactions, as developed in mechanically driven rotating flows (Kerswell 2002; Le Bars et al. 2015).

Rotating flows can become unstable through the so-called triadic resonance (Le Bars et al. 2015), i.e. a base flow with frequency $\omega_{0}$ and azimuthal wavenumber $m_{0}$ can

$\dagger$ Email address for correspondence: linyf@sustech.edu.cn 
excite two inertial waves whose frequencies $\omega_{1}, \omega_{2}$ and azimuthal wavenumbers $m_{1}, m_{2}$ match the resonance conditions (Bretherton 1964; Kerswell 2002): (Here we present the resonance conditions in a spherical geometry. In an unbounded domain, the second condition should read $\boldsymbol{k}_{1} \pm \boldsymbol{k}_{2}=\boldsymbol{k}_{0}$, where $\boldsymbol{k}$ is the wavevector.)

$$
\omega_{2} \pm \omega_{1}=\omega_{0}, \quad m_{2} \pm m_{1}=m_{0} .
$$

Such an instability has been observed both experimentally and numerically in a variety of rotating flows driven by mechanical forcings (see Kerswell 2002; Le Bars et al. 2015, and references therein), but never reported before in rotating convection systems. Motivated by geophysical and astrophysical applications, rotating convection has been studied extensively both for planar models (see recent review by Plumley \& Julien 2019) and for spherical models (e.g. Aubert et al. 2001; Christensen 2002; Gastine et al. 2016; Kaplan et al. 2017; Guervilly et al. 2019), yet the nonlinear dynamics and turbulent regime remain to be fully understood (Aurnou et al. 2015). Recent numerical simulations (Horn \& Schmid 2017) and laboratory experiments (Aurnou et al. 2018) of rotating convection in a cylinder using liquid gallium $(\operatorname{Pr} \approx 0.025)$ observed multi-modal interactions, yet the underlying mechanism remains to be elucidated. Lam et al. (2018) numerically studied rotating convection in a sphere with $\operatorname{Pr}=0.0023$ at moderate Ekman numbers and found that the nonlinear interaction of various thermal inertial waves leads to a weakly turbulent state, though no evidence of triadic resonances was shown. Here we show, through numerical simulations at lower Ekman numbers, that the primary convective mode at low $\operatorname{Pr}$ (relevant to liquid metals) can excite a pair of inertial modes through the triadic resonance in a rotating sphere, leading to more complex convective motions. Increasing the thermal forcing leads to a turbulent state that shows signatures of inertial waves. Our study may offer a new pathway to study rotating turbulent convection within the framework of inertial wave turbulence (Godeferd \& Moisy 2015; Le Reun et al. 2017).

\section{Numerical model}

We consider the canonical problem of Boussinesq convection in a sphere of radius $r_{o}$, which rotates at $\Omega=\Omega \hat{z}$. Convection is driven by a uniform heat source $S$ under a gravitational field $\boldsymbol{g}=-g_{o} \boldsymbol{r} / r_{o}$. The problem was first formulated by Chandrasekhar (1961) and is governed by the following dimensionless equations in the rotating frame:

$$
\begin{gathered}
E\left(\frac{\partial \boldsymbol{u}}{\partial t}+\boldsymbol{u} \cdot \boldsymbol{\nabla} \boldsymbol{u}\right)+\hat{\boldsymbol{z}} \times \boldsymbol{u}=-\nabla p+\frac{E R a}{P r} T \boldsymbol{r}+E \nabla^{2} \boldsymbol{u} \\
\operatorname{Pr}\left(\frac{\partial T}{\partial t}+\boldsymbol{u} \cdot \boldsymbol{\nabla} T\right)=\nabla^{2}\left(T-T_{b}\right), \\
\boldsymbol{\nabla} \cdot \boldsymbol{u}=0,
\end{gathered}
$$

where $\boldsymbol{u}$ is the fluid velocity, $p$ is the reduced pressure, $T$ is the total temperature and $T_{b}=\left(1-r^{2}\right) / 2$ is the dimensionless basic temperature without convection. The system is defined by three non-dimensional parameters, i.e. the Ekman number $E$, the Rayleigh number $R a$ and the Prandtl number Pr:

$$
E=\frac{\nu}{2 \Omega r_{o}^{2}}, \quad R a=\frac{\alpha g_{o} \beta r_{o}^{5}}{\nu \kappa}, \quad \operatorname{Pr}=\frac{\nu}{\kappa},
$$

where $\nu$ is the kinematic viscosity, $\kappa$ is the thermal diffusivity, $\alpha$ is the thermal expansion coefficient and $\beta=S /(3 \kappa)$ characterizes the temperature gradient without convection. 
In rotating flows, another important non-dimensional parameter is the Rossby number, which is defined as

$$
R o=\frac{U_{r m s}}{2 \Omega r_{o}} .
$$

In our model, the Rossby number $R o$ is an output parameter and is determined by the dimensional root mean square velocity $U_{r m s}$ in the simulation domain.

The fully nonlinear governing equations of the system are numerically solved using a spectral method based on expansions of spherical harmonics in the angular direction and Jones-Worland polynomials in the radial direction (Marti \& Jackson 2016). We use the no-slip boundary condition and a fixed-temperature $(T=0)$ condition on the spherical boundary. Simulations are initiated from small random velocity perturbations or from a saturated state at lower $R a$.

This study explores rotating convection in the parameter regime of low Ekman numbers $\left(10^{-6} \leqslant E \leqslant 10^{-5}\right)$ and low Prandtl numbers $\left(10^{-3} \leqslant \operatorname{Pr} \leqslant 10^{-2}\right)$. The Rayleigh number varies from the slightly supercritical to the turbulent convection regime. The resulting Rossby number ranges from $10^{-3}$ to $3 \times 10^{-2}$, suggesting that convection is rotationally dominated in all of the simulations. We use numerical truncations of $N=63$, $L=M=127$ for the simulations near the onset, and increase the truncations up to $N=191, L=M=383$ for the turbulent convection, where $N, L$ and $M$ are truncations of the radial polynomials, degree and order of spherical harmonics, respectively. The convergence of the numerical solutions is warranted by checking energy spectra and by doubling the truncations for some cases.

\section{Results}

Figure 1 shows the Fourier transform of the time series of the radial velocity at a fixed position $(r=0.59)$ in the equatorial plane for various $R a$ at $E=10^{-6}$ and $\operatorname{Pr}=0.001$. For $R a=1.5 \times 10^{7} \approx 1.4 R a_{c}$, where $R a_{c}$ is the critical Rayleigh number for the onset of convection, the frequency spectrum shows a pronounced peak at $\omega / \Omega=0.25$, which corresponds to an oscillatory convective mode at low $\operatorname{Pr}$ (Zhang 1994). In figure 1 (a), there is also an obvious peak at $\omega / \Omega \approx 0.5$, twice the frequency of the primary mode. Similar doubling in frequency has been observed in recent numerical simulations (Horn \& Schmid 2017) and laboratory experiments (Aurnou et al. 2018) using liquid metal in a rotating cylinder . As we shall show later, the doubling in frequency is accompanied by a doubling in the azimuthal wavenumber as well, which may result from the nonlinear selfinteraction of the primary mode. (The nonlinear term of a flow $\boldsymbol{u} \propto \mathrm{e}^{\mathrm{i}(\omega t+m \phi)}$ potentially has two components: $m_{0}=0, \omega_{0}=0$ and $\left.m_{2}=2 m, \omega_{2}=2 \omega\right)$. For $R a=1.7 \times 10^{7} \approx$ $1.6 R a_{c}$, apart from similar peaks observed in figure 1(a), the spectrum exhibits two distinct peaks with similar amplitudes at $\omega / \Omega=0.09$ and 0.16 , respectively ( figure $1(\mathrm{~b}))$. These two peaks and the primary peak at $\omega / \Omega=0.25$ satisfy the triadic resonance condition, i.e. $\omega_{1} \pm \omega_{2}=\omega_{0}$, suggesting a possible resonant triad between the primary convective mode and a pair of free modes.

Figure 2 shows the kinetic energy contained in different azimuthal wavenumbers $m$ for the case in figure 1(b). We can see from the time-averaged spectrum that the energy is dominated by the $m=4$ component, with subsequent major contributions from the $m=0,1,3$ and 8 components. Figure 2(b) shows the time evolution of the kinetic energy for the aforementioned components. After a transient stage, the $m=4$ component starts to grow and becomes the dominant one, which corresponds to the primary convective mode. As the primary mode becomes saturated, the $m=0$ and $m=8$ components also develop and saturate. The $m=0$ component represents a steady zonal flow, while 

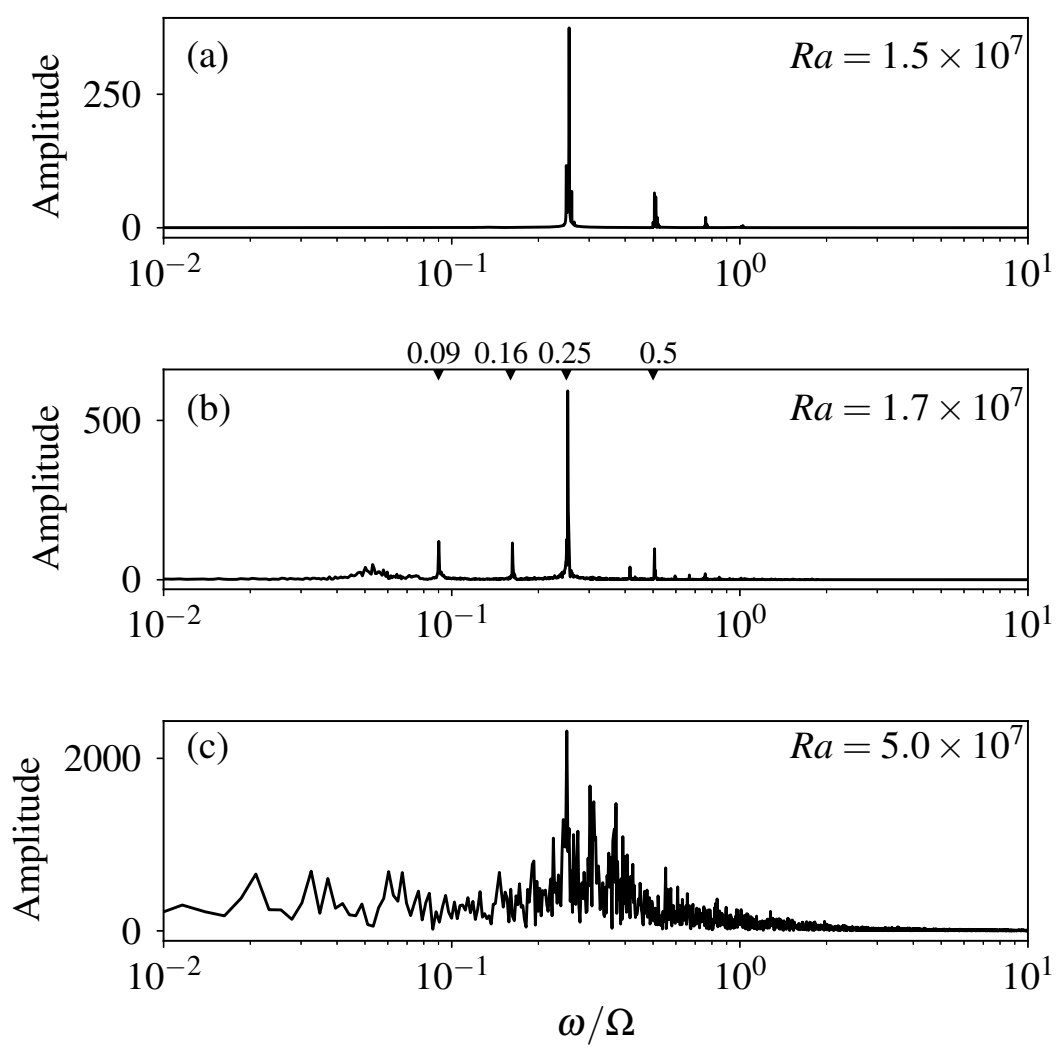

FiguRE 1. Fourier transform of the radial velocity $u_{r}$ at a fixed position $(r=0.59)$ in the equatorial plane at $E=10^{-6}$ and $\operatorname{Pr}=0.001$, but various $R a$. Frequencies are normalized by the rotation frequency $\Omega$.

$m=8$ is twice the azimuthal wavenumber of the primary mode. Both $m=0$ and $m=8$ components can be attributed to the nonlinear interaction of the convective mode with itself (Zhang \& Liao 2017). More interestingly, at $\Omega t /(2 \pi) \approx 1000$, the $m=1$ and $m=3$ components start to grow exponentially with the same growth rate and then saturate to similar levels, again pointing to a possible triadic resonance between the primary mode $m=4$ and two free modes of $m=1$ and $m=3$.

In order to confirm that both $\omega$ and $m$ simultaneously satisfy the triadic resonance conditions, we decompose the velocity field into different $m$ components and then analyse the time evolution of each component. Figure 3 shows the time evolution of the radial velocity along a fixed vertical line for $m=1,3,4$ and 8 components, respectively, for the case in figure 2. As the $m=0$ component represents a steady zonal flow, it is not shown in figure 3 . One can see that each $m$-component corresponds to a single frequency mode, and the $m=1,3,4$ modes have the simplest structure along the axis of rotation. We extract the frequencies of each mode by fitting the time series with a sinusoidal function and find $\omega / \Omega=0.1624,0.0899,0.2529$ for the $m=1,3,4$ modes respectively (see Table 1 ), which clearly satisfy the triadic resonance conditions in both $\omega$ and $m$. These results convincingly demonstrate that the primary convective mode with $m=4$ excites two free modes with $m=1$ and 3 through the triadic resonance. We also find that the $m=8$ mode has a frequency of $\omega / \Omega=0.5058$, doubling both $m$ and $\omega$ of the primary mode, as we mentioned before. 

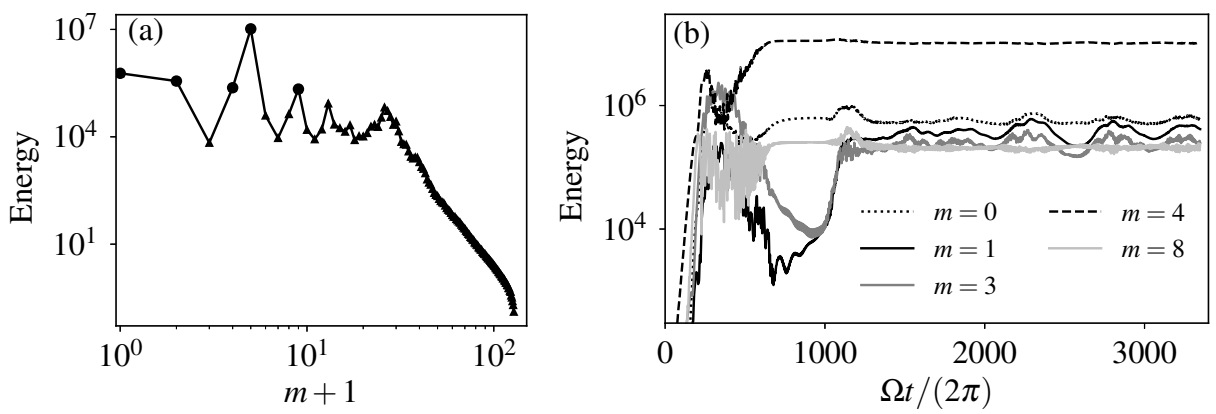

Figure 2. (a) Time-averaged kinetic energy spectrum as a function of the azimuthal wavenumber $m$ at $E=10^{-6}, \operatorname{Pr}=0.001$ and $R a=1.7 \times 10^{7}$. (b) Time evolution of the kinetic energy contained in different $m$, for the five largest components (represented by circles) in panel (a). Time is in the unit of the rotation period.

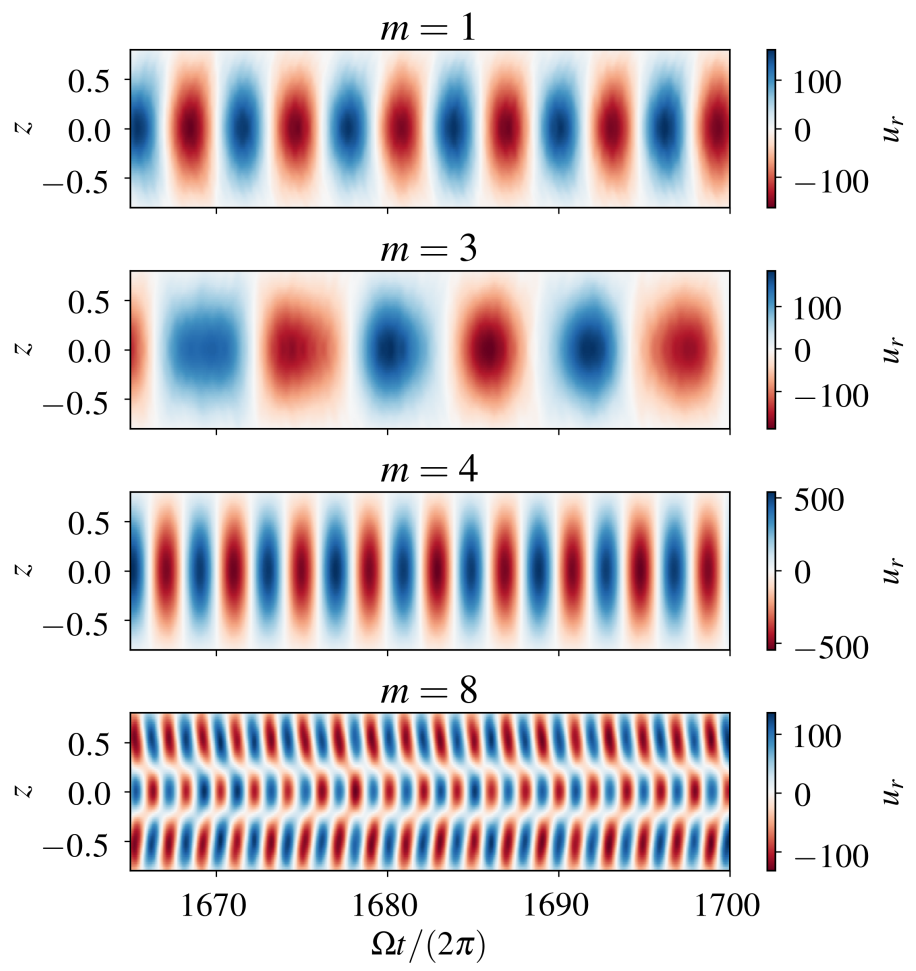

Figure 3. Time evolution of the radial velocity along a fixed vertical line with the distance to the rotation axis $s=0.5$ for the case in Fig. 2. The velocity field has been decomposed into components with different azimuthal wavenumber $m$.

Table 1 lists three examples of triadic resonances and involved modes (see also supplementary Movie 1, Movie 2, Movie 3 for the radial velocity of interacting modes in the equatorial plane) at different control parameters. We can see that the triadic resonance can excite modes with either lower or higher wavenumbers and frequencies with respect to the primary modes, providing a mechanism for the bi-directional energy transfer. For instance, at $E=10^{-5}, \operatorname{Pr}=0.01$ and $R a=3.3 \times 10^{6}$, a low-frequency $(\omega / \Omega=0.0918)$ 


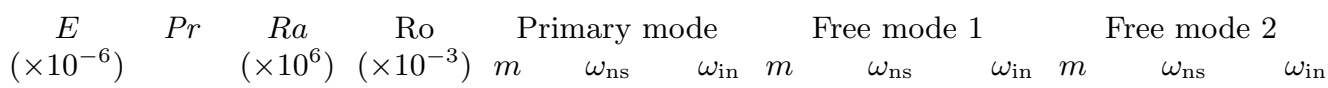

$$
\begin{aligned}
& \begin{array}{lllllllllllll}
1 & 0.001 & 17 & 2.4 & 4 & 0.2529 & 0.2613 & 1 & 0.1624 & 0.1766 & 3 & 0.0899 & 0.0715
\end{array}
\end{aligned}
$$

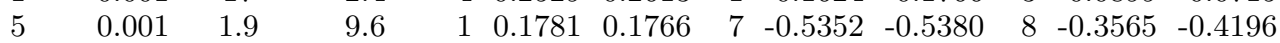

$$
\begin{aligned}
& \begin{array}{lllllllllllll}
10 & 0.01 & 3.3 & 2.2 & 3 & 0.0918 & 0.0715 & 10 & -0.3596 & -0.3771 & 13 & -0.2678 & -0.3303
\end{array}
\end{aligned}
$$

TABLE 1. Examples of the triadic resonance at different control parameters and involved modes. $\omega_{\text {ns }}$ is the frequency obtained from numerical simulations and $\omega_{\text {in }}$ is the frequency of purely inviscid inertial modes. Frequencies are in the unit of the rotation frequency and positive (negative) values correspond to prograde (retrograde) modes. For each case, a movie showing the radial velocity of the interacting modes in the equatorical plane is also provided.
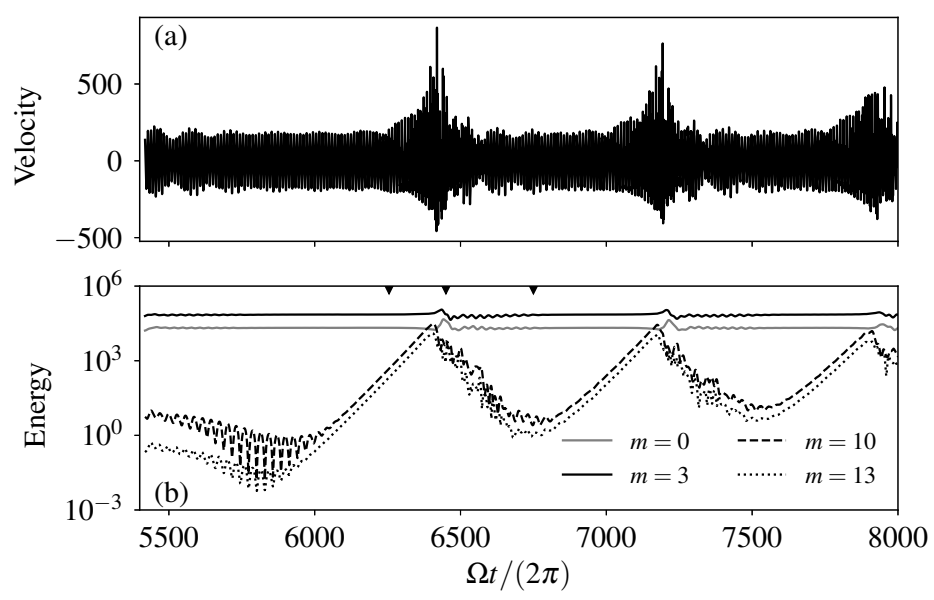

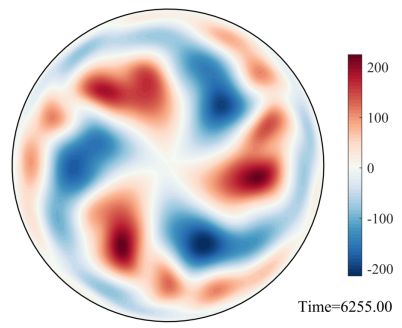

(c)

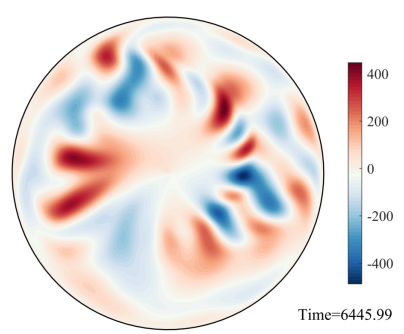

(d)

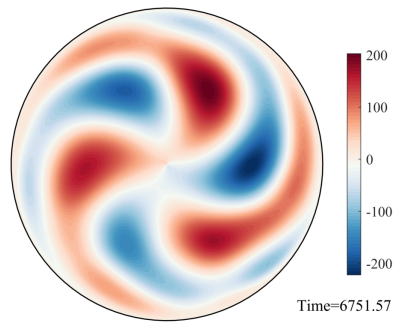

(e)

Figure 4. The growth and collapse of the unstable modes at $E=10^{-5}, \operatorname{Pr}=0.01$ and $R a=3.3 \times 10^{6}$. (a)Time series of the radial velocity at a fixed point. (b) Time series of the kinetic energy contained in different $m$ components; (c-e) Snapshots of the radial velocity in the equatorial plane at instances indicated by the inverted black triangles in panel (b).

prograde mode of $m=3$ excites a pair of retrograde modes with higher frequencies $(\omega / \Omega=0.3596$ and 0.2678$)$ and wavenumbers $(m=10$ and 13$)$. Note that the primary mode in this case shows a multi-cellular pattern of the radial structure (Net et al. 2008), whereas the primary mode in figure 3 shows a mono-cellular pattern of the radial structure (see supplementary movies). In this case, we also observe cyclic growth and collapse of the unstable modes (figure 4(a-b)), which is a typical behaviour of the triadic resonance (Lin et al. 2014; Barker 2016). We can see that higher-wavenumbers modes are excited on top 

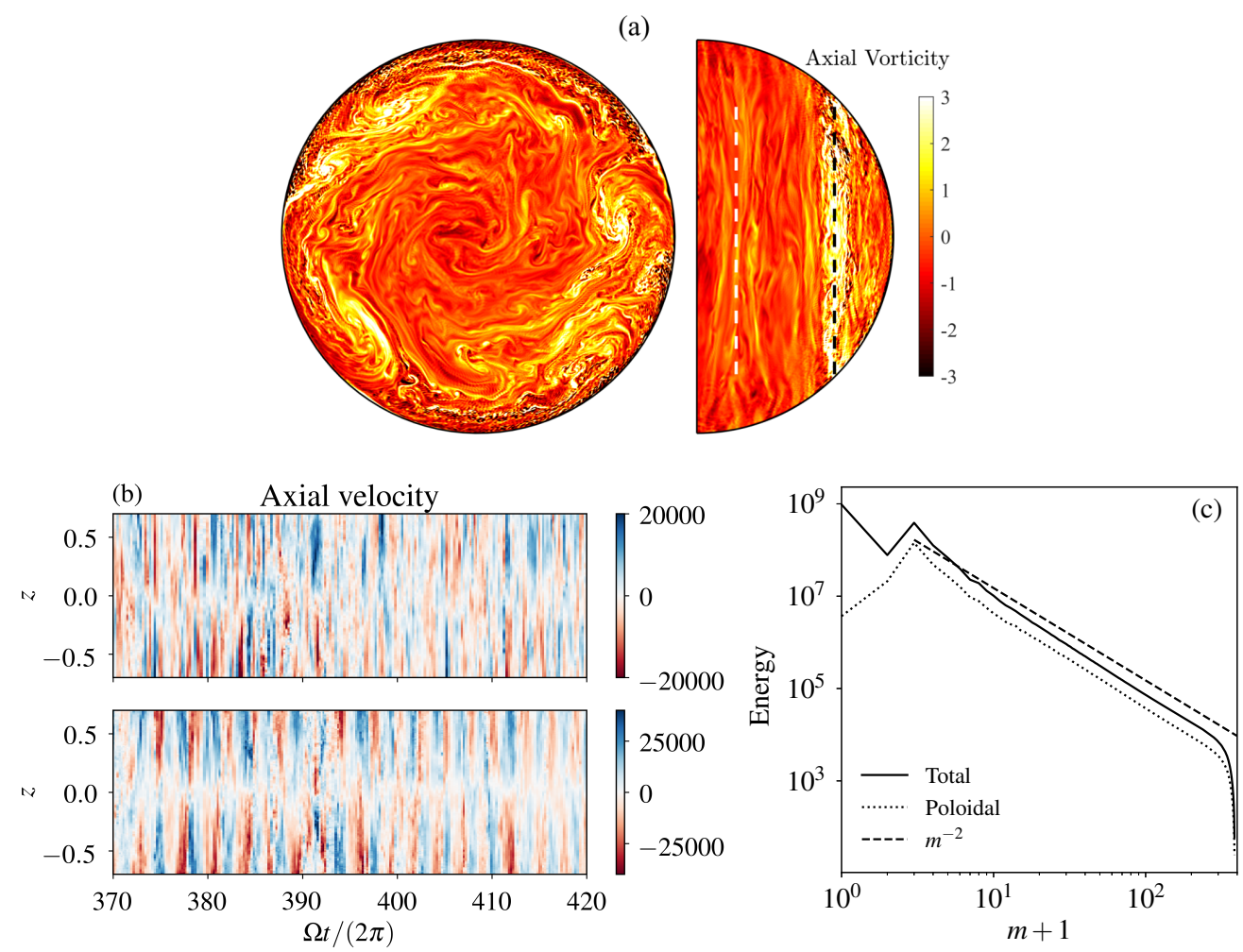

FiguRE 5. (a) A snapshot of the axial vorticity in the equatorial plane (left) and in a meridional plane (right); (b) Time evolution of the axial velocity along the white (top panel) and black (bottom panel) dashed lines in (a); (c) Energy spectra as a function of the azimuthal wavenubmer m. $E=10^{-6}, \operatorname{Pr}=0.001$ and $R a=5.0 \times 10^{7}$.

of the $m=3$ primary mode during the growth phase (figure 4(c)). As the energy in the growing modes becomes comparable to the zonal flow $(m=0)$, the flow suddenly collapses to chaotic small scales (figure 4(d)), known as resonant collapse of inertial modes, which was first observed by McEwan (1970) in a precessing cylinder. The collapse is followed by a relaminarization (figure $4(\mathrm{e})$, and then the cycle repeats. The whole process is reminiscent of the triadic resonant instability observed in several mechanically driven rotating flows (Le Bars et al. 2015, and references therein).

Table 1 also lists frequencies of purely inviscid inertial modes in a sphere which have similar spatial structures as the modes observed from numerical simulations. We note that the observed frequencies are slightly shifted from the inviscid frequencies due to the viscous effect, the detuning effect (Kerswell 2002) and the coupling with the thermal equation (Zhang 1994). Here we report a few cases in which the interacting modes can be clearly identified. For large $R a$ and low $E$, triadic resonances can simultaneously excite several pairs of modes, which makes it more difficult to identify individual modes. However, the mechanism appears to be generic as long as the onset of convection is in the form of an oscillatory thermal inertial mode, which generally exists at $\operatorname{Pr}<1$ (Zhang 1994).

Further increasing $R a$ leads to several modes interacting and eventually to a turbulent state. Figure 5 shows an example of turbulent flows at $E=10^{-6}, \operatorname{Pr}=0.001$ and $R a=5.0 \times 10^{7}$. The Rossby number in this case is calculated to be $R o=2.9 \times 10^{-2}$, 
suggesting that the turbulence convection is strongly influenced by the rotation. The axial vorticity (figure 5(a)) exhibits both small-scale and large-scale structures elongated along the rotation axis. These large-scale vortices located near the equator would merge into a single vortex at the centre when the stress-free boundary condition is used (Lin \& Jackson 2021). Figure 5(b) shows the time evolution of the axial velocity along two dashed lines in figure 5(a), which exhibits wave-like behaviours with typical frequencies within the spectrum of inertial waves (see also figure 1(c)). Figure 5(c) shows the energy spectrum as a function of the azimuthal wavenumber $m$. We can see from the spectrum that there is a dominant toroidal $m=0$ component, which represents a strong mean zonal flow. For smaller scales $(20 \lesssim m \lesssim 200)$, we see that the energy spectrum follows a power law of $m^{-2}$, which is reminiscent of the energy spectrum of inertial wave turbulence driven by elliptical instability (Le Reun et al. 2017). The power law $m^{-2}$ is also predicted by Zhou (1995) using a phenomenological approach for rotating turbulence. These observations suggest that the interaction of inertial waves plays an important role even in the turbulent regime, though further investigations are required in future.

\section{Conclusion}

We have shown for the first time that triadic resonances are taking place in a rotating convection system. The convective modes can excite either a pair of inertial waves with lower frequencies and wavenumbers or a pair with higher frequencies and wavenumbers, depending on the structure of the primary mode. This provides a possible mechanism for bi-directional energy transfer. The turbulent regime also shows signatures of inertial waves, and is reminiscent of inertial wave turbulence as observed in mechanically driven rotating flows. This study paves the way to study the nonlinear dynamics and turbulence in rotating convection within the framework of inertial wave interactions.

This study suggests that thermally (at low $P r$ ) and mechanically (e.g. tides, precession and libration) driven rotating flows share many features even in the nonlinear and turbulent regime, which may have significant implications for some fundamental problems in geophysical and astrophysical fluid dynamics. Both convection (Jones 2015) and mechanical forcings (Le Bars et al. 2015) are thought to be possible driven mechanisms for the generation of the Earth's and planetary magnetic fields through the dynamo process. It is possible that dynamos driven by two different mechanisms also share many features in certain parameter regimes and can be studied within the same framework. In stars and giant planets, the interaction between convection and tides is of great importance for calculating the efficiency of tidal dissipation, yet the problem remains poorly understood (Ogilvie 2014). Turbulent convection is usually treated as an effective viscosity in damping the tidal flow (Zahn 1989; Vidal \& Barker 2020). However, given small $\mathrm{Pr}$ in the convective zone of rotating stars and giant planets, both convection and tides can drive inertial waves, which would be interacting simultaneously and make it more difficult to estimate the efficiency of tidal dissipation in these systems.

In this paper, we consider rotating convection in a whole sphere. It is also of great interests to consider the problem in a spherical shell, in which behaviours of inertial waves are complicated by the critical latitudes and wave attractors (Rieutord et al. 2001). Therefore, interactions of thermal inertial waves in a spherical shell may exhibit more complex dynamics, which will be considered in future.

\section{Declaration of Interests}

The authors report no conflict of interest. 


\section{Supplementary movies}

Supplementary movies are available at Movie 1, Movie 2, Movie 3.

\section{Acknowledgements}

The author would like to thank Philippe Marti for providing the numerical code, Andy Jackson for comments on an early draft of this paper, Adrian Barker for useful discussions and the anonymous referees for their helpful comments. This study was supported by the B-type Strategic Priority Program of the CAS (XDB41000000), an NSFC grant (41904066), and the pre-research project on Civil Aerospace Technologies of CNSA (D020308) and the Macau Foundation. Simulations were performed on the Taiyi cluster supported by the Center for Computational Science and Engineering of Southern University of Science and Technology.

\section{REFERENCES}

Aldridge, K. \& Toomre, A. 1969 Axisymmetric inertial oscillations of a fluid in a rotating spherical container. J. Fluid Mech. 37, 307-323.

Aubert, J, Brito, D, Nataf, H.-C., Cardin, P \& Masson, J.-P. 2001 Asystematic experimental study of rapidly rotating spherical convection in water and liquid gallium. Phys. Earth Planet. Inter. 128, 51-74.

Aurnou, J.M., Calkins, M.A., Cheng, J.S., Julien, K., King, E.M., Nieves, D., Soderlund, K.M. \& Stellmach, S. 2015 Rotating convective turbulence in Earth and planetary cores. Phys. Earth Planet. Inter. 246, 52-71.

Aurnou, J. M., Bertin, V., Grannan, A. M., Horn, S. \& Vogt, T. 2018 Rotating thermal convection in liquid gallium: multi-modal flow, absent steady columns. J. Fluid Mech. 846, 846-876.

BARKER, A. J. 2016 Non-linear tides in a homogeneous rotating planet or star: global simulations of the elliptical instability. Mon. Not. R. Astr. Soc. 459 (1), 939-956.

Bretherton, F. P. 1964 Resonant interactions between waves. the case of discrete oscillations. J. Fluid Mech. 20 (3), 457-479.

Busse, F. H. 1970 Thermal instabilities in rapidly rotating systems. J. Fluid Mech. 44, 441-460.

Chandrasekhar, S. 1961 Hydrodynamic and hydromagnetic stability. Clarendon Press.

Christensen, U. $\sim$ R. 2002 Zonal flow driven by strongly supercritical convection in rotating spherical shells. J. Fluid Mech. 470, 115-133.

Gastine, T., Wicht, J. \& Aubert, J. 2016 Scaling regimes in spherical shell rotating convection. J. Fluid Mech. 808, 690-732.

Godeferd, F. S. \& Moisy, F. 2015 Structure and dynamics of rotating turbulence: A review of recent experimental and numerical results. Appl. Mech. Rev. 67 (3), 1-13.

Greenspan, H. P. 1968 The Theory of Rotating Fluids. London: Cambridge University Press.

Guervilly, C., Cardin, P. \& Schaeffer, N. 2019 Turbulent convective length scale in planetary cores. Nature $\mathbf{5 7 0}$ (7761), 368-371.

Hollerbach, R. \& Kerswell, RR 1995 Oscillatory internal shear layers in rotating and precessing flows. J. Fluid Mech. 298, 327-339.

Horn, S. \& Schmid, P. J. 2017 Prograde, retrograde, and oscillatory modes in rotating Rayleigh-Bénard convection. J. Fluid Mech. 831, 182-211.

Jones, C.A. 2015 Thermal and Compositional Convection in the Outer Core. In Treatise on Geophysics, , vol. 8, pp. 115-159. Elsevier.

Jones, Chris A., Soward, Andrew M. \& Mussa, Ali I. 2000 The onset of thermal convection in a rapidly rotating sphere. J. Fluid Mech. 405 (2000), 157-179.

Kaplan, E. J., Schaeffer, N., Vidal, J. \& Cardin, P. 2017 Subcritical Thermal Convection of Liquid Metals in a Rapidly Rotating Sphere. Phys. Rev. Lett. 119 (9), 094501.

Kerswell, R. R. 2002 Elliptical instability. Annu. Rev. Fluid Mech. 34, 83-113. 
Lam, Kameng, Kong, Dali \& Zhang, Keke 2018 Nonlinear thermal inertial waves in rotating fluid spheres. Geophys. Astrophys. Fluid Dyn. 112 (5), 357-374.

Le Bars, M., Cébron, D. \& Le Gal, P. 2015 Flows Driven by Libration, Precession, and Tides. Annu. Rev. Fluid Mech. 47 (1), 163-193.

Le Reun, T., Favier, B., Barker, A. J. \& Le Bars, M. 2017 Inertial Wave Turbulence Driven by Elliptical Instability. Phys. Rev. Lett. 119 (3), 034502.

Lin, Y. \& JACKSON, A. 2021 Large-scale vortices and zonal flows in spherical rotating convection. J. Fluid Mech. In press.

Lin, Y., Noir, J. \& JACKson, A. 2014 Experimental study of fluid flows in a precessing cylindrical annulus. Phys. Fluids 26 (4), 046604.

MARTi, P. \& JACKSON, A. 2016 A fully spectral methodology for magnetohydrodynamic calculations in a whole sphere. J. Comput. Phys. 305, 403-422.

McEwAn, A. D. 1970 Inertial oscillations in a rotating fluid cylinder. J. Fluid Mech. 40 (03), 603.

Net, Marta, Garcia, Ferran \& Sánchez, Juan 2008 On the onset of low-Prandtl-number convection in rotating spherical shells: Non-slip boundary conditions. J. Fluid Mech. 601, $317-337$.

Noir, J., Brito, D., Aldridge, K. \& Cardin, P. 2001 Experimental evidence of inertial waves in a precessing spheroidal cavity. Geophys. Res. Lett. 28 (19), 3785-3788.

Ogilvie, G. I. 2014 Tidal Dissipation in Stars and Giant Planets. Annu. Rev. Astron. Astrophys. $\mathbf{5 2}, 1-46$.

Ogilvie, G. I. \& Lin, D. N. C. 2004 Tidal Dissipation in Rotating Giant Planets. Astrophys. J. 610 (1), 477-509.

Plumley, M. \& Julien, K. 2019 Scaling Laws in Rayleigh-Bénard Convection. Earth and Space Science 6 (9), 1580-1592.

Rieutord, M., Georgeot, B. \& Valdettaro, L. 2001 Inertial waves in a rotating spherical shell: attractors and asymptotic spectrum. J. Fluid Mech. 435, 103-144.

Spiegel, E A 1971 Convection in Stars I. Basic Boussinesq Convection. Annu. Rev. Astron. Astrophys. 9 (323).

VIDAL, J. \& BARKER, A. J. 2020 Efficiency of tidal dissipation in slowly rotating fully convective stars or planets. Mon. Not. R. Astr. Soc. 497 (4), 4472-4485.

ZAHN, J. P. 1989 Tidal evolution of close binary stars. I - Revisiting the theory of the equilibrium tide. Astr. Astrophys. 220 (1-2), 112-116.

ZhANG, K. 1994 On coupling between the Poincaré equation and the heat equation. J. Fluid Mech. 268, 211.

Zhang, K. \& LiaO, X. 2017 Theory and Modeling of Rotating Fluids: convection, inertial waves, and precession. Cambridge: Cambridge University Press.

Zhou, Y. 1995 A phenomenological treatment of rotating turbulence. Phys. Fluids 7 (8), 20922094. 